\title{
Logical Form of Complex Sentences in Task-Oriented Dialogues*
}

\author{
Cecile T. Balkanski \\ Harvard University, Aiken Computation Lab \\ Cambridge, MA 02138
}

\section{Introduction}

Although most NLP researchers agree that a level of "logical form" is a necessary step toward the goal of representing the meaning of a sentence, few people agree on the content and form of this level of representation. An even smaller number of people have considered the complex action sentences that are often expressed in taskoriented dialogues. Most existing logical form representations have been developed for single-clause sentences that express assertions about properties or actual actions and in which time is not a main concern. In contrast, utterances in task-oriented dialogues often express unrealized actions, e.g., (1a), multiple actions and relations between them, e.g., (1b), and temporal information, e.g., (1c):

(1) a. What about rereading the Operations manual?

b. By getting the key and unlocking the gate, you get ten points.

c. When the red light goes off, push the handle.

In the following sections, I discuss the issues that arise in defining the logical form of these three types of sentences. The Davidsonian treatment of action sentences is the most appropriate for my purposes because it treats actions as individuals [7]. For example, the logical form of "Jones buttered the toast" is a three place predicate, including an argument position for the action being described, i.e., $\exists x$ butter (jones, toast, $x$ ). The presence of the action variable makes it possible to represent optional modifiers as predications of actions and to refer to actions in subsequent discourse. Furthermore, and more importantly for the present purpose, it facilitates the representation of sentences about multiple actions and relations between them.

\section{Unrealized-action sentences}

A Davidsonian logical form of sentence (1a), namely $\exists x \operatorname{reread}(u s$, manual, $x)$, makes the claim that there exists a particular action $x$. But this is not the intended meaning of the sentence. Instead, this sentence concerns a hypothetical action. The same problem arises with sentences (1b) and (1c) which state how typical actions are related or when to perform a future action. Apparently, Davidson did not have these types of action in mind when suggesting his theory of logical form.

In fact, a closer look at the literature shows that the problem of representing action sentences that do

*This research has been supported by U S West Advanced Technologies, by the Air Force Office of Scientific Research under Contract No.AFOSR-89-0273, and by an IBM Gradnate Fellowship. not make claims about actions that have or are occurring (i.e., actual actions) has been virtually ignored. Hobbs, who also adopts a Davidsonian treatment of action sentences, is one notable exception [11]. His "Platonic universe" contains everything that can be spoken of and the predicate Exist is used to make statements about the existence in the actual universe of individuals in the Platonic universe. For example, the formula Exists $(x) \wedge r_{u n}(x, j o h n)$ says that the action of John's running exists in the actual universe, or, more simply, that John runs. The approach I am currently investigating is to extend Hobbs' representation by introducing predicates stating the existence of actions in future, hypothetical or typical worlds as well as in the actual world.

Another possibility is to adopt the standard philosophical approach to the representation of properties, and for that matter, of actions, that are not actually instantiated, namely possible worlds (cf. $[13,2]$ ). Furthermore, and independently of the approach that is adopted, there is a need to identify the different types of unrealized actions and determine whether they should be distinguished in the logical form.

\section{Multi-clause sentences}

Another area of logical form that has not received much attention is the representation of sentences about multiple actions and relations between them. I have been investigating sentences including by- and to- purpose clauses because they are used to communicate two action relations, namely generation and enablement, which I have defined elsewhere [3]. In a Davidsonian logical form, the connectives "by" and "to" can be represented as two-place predicates ranging over action tokens ${ }^{1}$; e.g.:

(2) To learn how to use the system, read the manual. learn $\left(y o u\right.$, system, $\left.x_{1}\right) \wedge \operatorname{read}\left(y o u\right.$, manual, $\left.x_{2}\right) \wedge$ inorderto $\left(x_{2}, x_{1}\right)$

Clauses may also be joined with coordination conjunctions, e.g., (3a), and the resulting constituent may participate in another action relation, as in (1b) and repeated below in (3b). I therefore represent these connectives by a three place predicate, e.g., and $\left(x_{1}, x_{2}, x_{4}\right)$ which is true if action $x_{4}$ is the conjunction of actions $x_{1}$ and $x_{2}$. In (3a), the action token $x_{4}$ might seem superfluous, but note that it becomes necessary if that action is referred to in subsequent discourse (e.g., "Do

\footnotetext{
${ }^{1}$ Although this problem interacts with the one discussed in the previous section, for the purpose of this presentation, I call Davidson's action variables action tokens and represent them as constants in the logical form.
} 
it fast!"); in (3b), the action token $x_{4}$ can then be used as the first argument to the by predicate:

(3) a. Get the key and unlock the gate. get $\left(\right.$ you, key, $\left.x_{1}\right) \wedge$ unlock $\left(\right.$ you, gate, $\left.x_{2}\right) \wedge$ and $\left(x_{1}, x_{2}, x_{4}\right)$

b. By getting the key and unlocking the gate, you get ten points.

get $\left(\right.$ you, key, $\left.x_{1}\right) \wedge$ unlock $\left(\right.$ you, gate, $\left.x_{2}\right) \wedge$ get $\left(\right.$ you, 10points, $\left.x_{3}\right) \wedge$ and $\left(x_{1}, x_{2}, x_{4}\right) \wedge b y\left(x_{4}, x_{3}\right)$

In the above logical forms, I assume that the $b y$ and inorderto predicates denote a two place relation expressing the "ideal meaning" of the corresponding English connective [9]. There is not necessarily a one-to-one mapping between particular linguistic expressions and action relations, and subsequent pragmatic processing of the logical forms will further interpret these relations.

Representing the embedded clause as an additional argument to the predicate representing the matrix clause (e.g., [5]), or representing the relation as a binary sentential operator (e.g., [16]) are alternative representations, both of which suffer from problems discussed by Davidson because action tokens become irrelevant. Furthermore, the first does not capture the intuitive notion that these sentences express action relations, and the second introduces a lack of homogeneity between logical forms of sentences involving action relations and those that do not.

\section{Time}

Still another feature that has been overlooked in the study of logical form is time. Although a number of papers include time in their logical forms, most do not discuss their treatment of time and consider primarily past and present tense examples about actual actions (e.g., $\left.[1,5,14]^{2}\right)$. The lack of concern for temporal issues is also characteristic of the literature on semantic interpretation (e.g., $[10,15,16])$. On the other hand, there is a vast literature on the interpretation and representation of tense, aspect and temporal modifiers, but these papers do not describe the logical forms from which their representations are generated (e.g., $[4,6,8,12,17,18])$.

Clearly, there is a missing link between the literature on logical form and that on tense and aspect. Providing such a link is one of the goals of this research. Davidson's treatment of action sentences does not provide a fully satisfying starting point. Although his initial paper does not include any example of temporal modifiers, he would probably represent them as predicates over action tokens, e.g., next_week $(x)$, a representation that does not make explicit reference to time (to which anaphors might refer). Introducing a time predicate, e.g., time $(x$, next_week), solves this particular problem, but introduces other complexities because this predicate would not be adequate for all temporal modifiers (e.g., compare Sue will leave in two hours and Sue reached the top in two hours). Given that the aspectual type and tense of the verb, along with the presence of adverbials and common sense knowledge all interact in the interpretation of the temporal information in a sentence [12], it might be preferable for such reasoning to be performed with the logical form as input rather than as output.

\footnotetext{
${ }^{2}$ Moore [14] addresses time issues, but omits future tense sentences and acknowledges problematic interactions between his event abstraction operator and time.
}

\section{Conclusion}

Although many researchers have proposed formalisms for simple action sentences, very few of them have addressed the issues that arise when extending those formalisms to the more complex sentences that occur in task-oriented dialogues. There has been work in each of the above areas, but this research has been fragmentary and still needs to be integrated with that on the logical form of action sentences. Ironically, the conclusion that Moore arrived at, ten years ago, is still valid today [14]: "If real progress is to be made on understanding the logical form of natural-language utterances, it must be studied in a unified way and treated as an important research problem in its own right." In my talk, I will present an initial attempt to do so.

\section{References}

[1] H. Alshawi \& J. van Eijck. Logical form in the core language engine. Proceedings of the $A C L, 1989$.

[2] D. Appelt. Planning English referring expressions. Artificial Intelligence 26, 1985.

[3] C. Balkanski. Modelling act-type relations in collaborative activity. Technical Report TR-23-90, Harvard University, 1990.

[4] M. Brent. A simplified theory of tense representations and constraints on their composition. Proceedings of the $A C L, 1990$.

[5] L. Creary. NFLT: A language of thought for reasoning about actions, 1983. working paper.

[6] M. Dalrymple. The interpretation of tense and aspect in English. Proceedings of the ACL, 1988.

[7] D. Davidson. The logical form of action sentences. In N. Rescher (ed), The Logic of Decision and Action. University Pittsburgh Press, 1967.

[8] M. Harper \& E. Charniak. Time and tense in english. Proceedings of the ACL, 1986.

[9] A. Herskovits. Language and Spatial Cognition. Cambridge University Press, 1986.

[10] G. Hirst. Semantic interpretation and ambiguity. Artificial Intelligence, 34, 1988.

[11] J. Hobbs. Ontological promiscuity. Proceedings of the $A C L, 1985$.

[12] M. Moens \& M. Steedman. Temporal ontology and temporal reference. Computational Linguistics, 14(2), 1988.

[13] R. Moore. A formal theory of knowledge and action. In J. Hobbs \& R. Moore (eds), Formal Theories of Commonsense Word. Ablex, 1985.

[14] R. Moore. Problems in logical form. Proceedings of the $A C L, 1981$.

[15] M. Pollack \& F. Pereira. An integrated framework for semantic and pragmatic interpretation. Proceedings of the $A C L, 1988$.

[16] L. Schubert \& F Pelletier. From English to logic: Contex-free computation of 'conventional' logical translations. Computional Linguistics, 10, 1984.

[17] B. Webber. Tense as discourse anaphor. Computational Linguistics, 14(2), 1988.

[18] K. Yip. Tense, aspect, and the cognitive representation of time. Proceedings of IJCAI, 1985. 